\title{
OPTIMAL EXPONENT HEAT BALANCE AND REFINED INTEGRAL METHODS APPLIED TO STEFAN PROBLEMS
}

\author{
T. G. MYERS
}

\begin{abstract}
When using a polynomial approximating function the most contentious aspect of the Heat Balance Integral Method is the choice of power of the highest order term. In this paper we employ a method recently developed for thermal problems, where the exponent is determined during the solution process, to analyse Stefan problems. This is achieved by minimising an error function. The solution requires no knowledge of an exact solution and generally produces significantly better results than all previous HBI models. The method is illustrated by first applying it to standard thermal problems. A Stefan problem with an analytical solution is then discussed and results compared to the approximate solution. An ablation problem is also analysed and results compared against a numerical solution. In both examples the agreement is excellent. A Stefan problem where the boundary temperature increases exponentially is analysed. This highlights the difficulties that can be encountered with a time dependent boundary condition. Finally, melting with a time-dependent flux is briefly analysed without applying analytical or numerical results to assess the accuracy.
\end{abstract}

\section{NOMENClATURE}

$E_{n}(t) \quad$ Least squares error

$e_{n} \quad E_{n}(t)=e_{n} t^{\alpha}$ error measure

$n \quad$ Exponent in approximating polynomial

$s(t) \quad$ Position of melt front

$t_{1} \quad$ Time when ablation begins

$u(x, t)$ Temperature

$\beta \quad$ Inverse Stefan number

$\delta(t) \quad$ Heat penetration depth

$\lambda \quad$ Growth rate $s=2 \lambda \sqrt{t}$

\section{INTRODUCTION}

The Heat Balance Integral Method (HBIM) was developed by Goodman, see $[7,12]$, to find approximate solutions to the heat equation and Stefan problems. In fact it is an adaptation of the earlier method of von Kárman and Pohlhausen for analysing boundary layer flow [24]. Since there are many exact solutions to the heat equation the HBIM has probably had the greatest impact on Stefan problems, where very few analytical solutions exist. 
The HBIM is primarily employed in heat transfer problems. However, obviously it may be applied to diffusion and porous media problems. The heat equation arises in viscous flow when a plate is impulsively moved in a semiinfinite viscous fluid. It is used in probability and describes random walks. It is also applied in financial mathematics for this reason and is a particular limit of the famous Black-Scholes equation. It is important in Riemannian geometry and thus topology, see for example $[6,21]$. The Schrödinger equation is related to the heat equation but has a complex diffusivity. The HBIM has recently been applied to modelling the temperature in a thermistor [10], the process of contact melting [19], the Korteweg-de-Vries equation [18] and the ignition time of wood [25]. A much more comprehensive list is given by Hristov [9]. Any improvement in the method can therefore have far reaching implications.

When analysing the standard thermal problem of heating a semi-infinite material occupying $x \geq 0$ and initially at a constant temperature the HBIM involves three steps:

(1) First the heat penetration depth, $\delta(t)$ is introduced. For $x \geq \delta$ the temperature change from the initial temperature is negligible.

(2) An approximating function, typically a polynomial, is introduced. This describes the temperature for $0 \leq x \leq \delta(t)$.

(3) The heat equation is integrated over $x \in[0, \delta]$ to produce what is termed the heat balance integral. This is solved to determine $\delta(t)$ and hence the temperature.

The result of this process is to reduce the governing heat equation to a single ordinary differential equation for $\delta$, which may often be solved analytically. Note, this final stage, to determine $\delta$, means that the heat equation is only solved in an integral sense [2]. That is, the choice of $\delta$ ensures that the area under $u_{t}$ and $u_{x x}$ match. This means that $u_{t}$ can be very different to $u_{x x}$ and so it is no surprise that certain choices of $u$ perform significantly better than others. Consequently there is much debate over, and variety in, the choice of approximating function. Goodman initially proposed a quadratic, but also briefly mentioned a cubic. Wood [26] showed that even for the quadratic there are six distinct formulations and the one employed by Goodman is generally only the third most accurate. In fact there is a seventh formulation, see [12]. Antic \& Hill [2] employ a cubic profile in the study of diffusion in grain silos. Myers et al [20] investigate the melting of a finite thickness block. A pre-melting solution motivates their choice of a cubic polynomial. Mitchell \& Myers [13] use a pre-melting solution to motivate a quartic approximation to the temperature in a study of ablation. A distinct modification to the method, known as the Refined Integral Method (RIM), was developed in $[22,23]$. This involves changing step 3 of the HBIM to evaluating a double integral. This method is discussed in more detail later.

With the correct scaling the standard approximating polynomials may be written in the form [9]:

$$
u=a_{n}\left(1-\frac{x}{\delta}\right)^{n} .
$$

Most authors assume $n$ to be an integer. Braga \& Mantelli $[3,4]$ took the next logical step and allowed $n$ to be a non-integer. For an ablation problem they chose $n$ based on matching the time to melting predicted by the exact solution and the 
approximate one. After melting they increase $n$ to give better agreement with a numerical solution. In fact in [13] it is shown that increasing $n$ leads to physically unrealistic results; for a small time the ablated material returns. Hristov [9] has carried out a detailed study of HBIM problems with a temperature of the form (1) and also provides a comprehensive review of previous work. He introduces two alternative constraints to determine $n$. The first requires matching the flux at $x=0$ with that of an exact solution. The second matches the integral of the exact and approximate temperatures. In both cases, and for a fixed temperature or fixed flux boundary condition, non-integer values of $n$ are found. Hristov states that 'the results oscillate around $n=3$ '. This ties in with the fact that the majority of studies take $n \in[2,4]$. In a separate paper Hristov [8] uses an entropy minimization technique that also involves matching at $x=0$ and so the results reduce to those of the previous paper.

This discussion obviously leads to the question, what is the best choice of $n$ ? All the aforementioned works take $n$ based on a knowledge of exact or numerical solutions, which then limits the applicability of the method. The accuracy of the method can then usually be measured by comparison with the known solution. In effect this makes the method redundant; if the exact or numerical solution is already known, why look for an approximate one?

Langford [11] allowed progress to be made on one front by introducing a definition for the error, and hence a measure of the accuracy, which does not require a known solution:

$$
E_{n}(t)=\int_{0}^{\delta}\left[\frac{\partial u}{\partial t}-\frac{\partial^{2} u}{\partial x^{2}}\right]^{2} d x \geq 0 .
$$

If $u$ is an exact solution to the heat equation then obviously $E_{n}=0$ for all time. Approximate solutions will have $E_{n}>0$. Taking the square of $u_{t}-u_{x x}$ prevents the cancelling of errors of opposite sign (this cancelling is the Achilles heel of the HBIM) and also magnifies the importance of regions where $u$ does not closely satisfy the heat equation [11]. Langford studied the problem where the temperature at $x=0$ is fixed and found $E_{n}=e_{n} t^{-3 / 2}$, where $e_{n}$ depends on $n$. In the following work we will employ (2) in a different way. We will treat $n$ as an unknown, rather than specifying it from the start, and then use (2) to provide an extra equation to determine $n$.

We will first illustrate the new method by applying it to a standard problem of heating a semi-infinite material. We will then extend the method to the corresponding Stefan problem, where melting occurs due to this heating and then give examples of ablation, travelling wave and time-dependent flux Stefan problems. In the travelling wave example the temperature at $x=0$ increases exponentially and the results demonstrate that the method is only applicable for small times.

\section{StANDARD THERMAL PROBLEMS}

2.1. Fixed temperature boundary condition. To introduce the optimal exponent method we now analyse what is perhaps the simplest thermal problem defined on a semi-infinite media, namely that of a material initially at a constant temperature which, at $t=0$, is heated to a different temperature on the boundary 
$x=0$, see $[6, \mathrm{p} 58]$. This problem may be expressed by

$$
\frac{\partial u}{\partial t}=\frac{\partial^{2} u}{\partial x^{2}} \quad u(0, t)=\left.1 \quad u\right|_{x \rightarrow \infty} \rightarrow 0 \quad u(x, 0)=0 .
$$

The exact solution is

$$
u=\operatorname{erfc} \frac{x}{2 \sqrt{t}}
$$

As discussed in the introduction, to apply the HBIM or RIM we must first introduce a function, known as the heat penetration depth, $\delta(t)$. For $x \geq \delta$ the temperature change above the initial value is negligible (although we do not define what constitutes negligible). For this example the heat penetration depth is defined by $u(\delta, t)=0$. For smoothness we also invoke $u_{x}(\delta, t)=0$. If necessary a further condition can be obtained by noting

$$
\frac{d u}{d t}(\delta, t)=\left.\left(\frac{\partial u}{\partial x} \frac{d \delta}{d t}+\frac{\partial u}{\partial t}\right)\right|_{x=\delta}=0 \quad \Rightarrow \quad \frac{\partial^{2} u}{\partial x^{2}}(\delta, t)=0,
$$

see [13]. In fact any number of similar conditions may be defined by successive differentiation of the boundary conditions. Subject to the above boundary conditions the appropriate approximating polynomial then takes the form (1) where $a_{n}=1$.

Finally we calculate the Heat Balance Integral. The standard HBIM involves integrating the heat equation over the region $x \in[0, \delta]$. Since $u(\delta, t)=u_{x}(\delta, t)=0$ this leads to

$$
\frac{\mathrm{d}}{\mathrm{d} t} \int_{0}^{\delta} u \mathrm{~d} x=-\left.\frac{\partial u}{\partial x}\right|_{x=0} .
$$

Substituting for $u$ from equation (1) gives a single ordinary differential equation for $\delta$ with solution

$$
\delta=\sqrt{2 n(n+1) t}
$$

where we have applied $\delta(0)=0$.

The RIM formulation involves integrating the heat equation twice with respect to $x$

$$
\int_{0}^{\delta}\left(\int_{0}^{x} \frac{\partial u}{\partial t} \mathrm{~d} \xi\right) \mathrm{d} x=\int_{0}^{\delta}\left(\frac{\partial u}{\partial x}-\left.\frac{\partial u}{\partial x}\right|_{x=0}\right) \mathrm{d} x
$$

After some manipulation this leads to the main equation for the RIM

$$
\frac{\mathrm{d}}{\mathrm{d} t} \int_{0}^{\delta} x u d x=u(0, t)
$$

for further details see $[12,16,22]$. Substituting for $u$ we find an equation for the heat penetration depth, which has the solution

$$
\delta=\sqrt{(n+1)(n+2) t} .
$$


In both methods we are left with a function $\delta(t)=f(n) \sqrt{t}$, which depends on the choice of the unknown exponent $n$. To determine this we examine the error defined by (2). This requires expressions for the two derivatives

$$
\frac{\partial u}{\partial t}=\frac{n x \delta_{t}}{\delta^{2}}\left(1-\frac{x}{\delta}\right)^{n-1} \quad \frac{\partial^{2} u}{\partial x^{2}}=\frac{n(n-1)}{\delta^{2}}\left(1-\frac{x}{\delta}\right)^{n-2} .
$$

Then evaluating the integral gives

$$
E_{n}(t)=\frac{n^{2}(2 n+1)\left[(2 n-1)(n-1)^{2}-\delta \delta_{t}(2 n-3)\right]+\delta^{2} \delta_{t}^{2} n(2 n-3)}{\delta^{3}(2 n-3)\left(4 n^{2}-1\right)}
$$

This is the function we must minimise to determine $n$. Substituting for $\delta$ for the HBIM we find

$$
E(t)=\frac{\left(6 n^{2}+2 n^{4}+2 n-7 n^{3}-1\right) \sqrt{2 n(n+1)}}{4(n+1)^{2}(2 n-3)\left(4 n^{2}-1\right)} t^{-3 / 2}=e_{n} t^{-3 / 2}
$$

where $e_{n}$ depends solely on $n$. So our choice of $n$ is based on minimizing $e_{n}$. For the RIM the error is

$$
\begin{aligned}
E(t) & =\frac{n\left(10 n^{5}-39 n^{4}+34 n^{3}+27 n^{2}-20 n-12\right) \sqrt{(n+1)(n+2)}}{4(2 n-3)\left(4 n^{2}-1\right)(n+1)^{2}(n+2)^{2}} t^{-3 / 2} \\
& =e_{n} t^{-3 / 2}
\end{aligned}
$$

so again we look for a minimum of $e_{n}$.

To satisfy the condition $u_{x x}(\delta, t)=0$ requires $n \geq 2$, in which case there is a single minimum of $e_{n}$ for each formulation. For the HBIM we find a minimum of $e_{n} \approx 0.0169$ for $n \approx 2.235$. the solution then asymptotes to 0.088 as $n \rightarrow \infty$. The minimum for the RIM formulation occurs for very similar $n \approx 2.218$ with a marginally smaller error, $e_{n} \approx 0.0167$ and an asymptote of 0.312 . Note, in both cases, $n \in[2,3]$ clarifying the popularity of the choices $n=2$ or 3 .

On Figure 1 we show a comparison of the exact solution against the approximate temperature from the HBIM and RIM formulations at $t=1$. This means that for the HBIM we set $n=2.235$, use this value to calculate $\delta \approx 3.802 \sqrt{t}$ from equation (7) and $u$ is then given by (1). For the RIM we choose $n=2.218$ and use $(10)$ to calculate $\delta \approx 3.684 \sqrt{t}$. The approximate curves show excellent agreement with the exact solution. Since $e_{n}$ is independent of time the minimum value of $n$ will remain fixed, and the actual value of $E_{n} \sim t^{-3 / 2}$ will decrease; so we expect this excellent agreement to continue for all time (this has been confirmed by testing the solutions for a wide range of times $\left.t \in\left[10^{-4}, 10\right]\right)$.

2.2. Constant flux and Newton cooling boundary conditions. If we change the boundary condition at $x=0$ to $u_{x}(0, t)=-1$, that is a constant flux condition then the approximate temperature has the form

$$
u=\frac{\delta}{n}\left(1-\frac{x}{\delta}\right)^{n}
$$

For the HBIM we find

$$
\delta=\sqrt{n(n+1) t}
$$




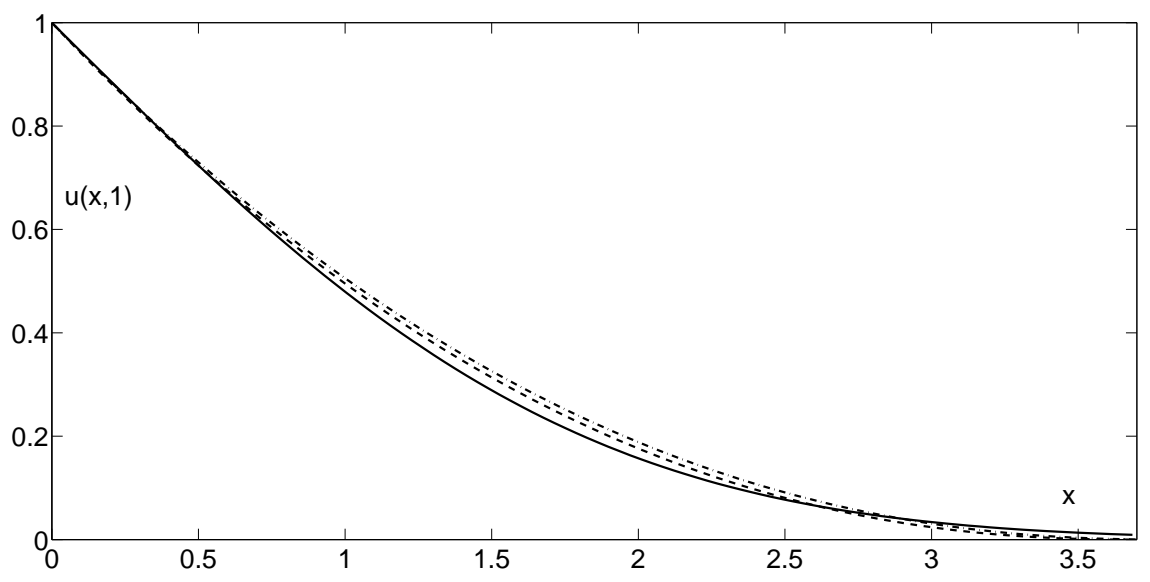

Figure 1. Plot of $u(x, t)$ at $t=1$. The exact solution is shown as a solid line, the HBIM solution with $n=2.235$ (dash-dot) and the RIM solution with $n=2.218$ (dashed)

whilst for RIM

$$
\delta=\sqrt{2(n+1)(n+2) t / 3} .
$$

The error integral becomes

(18)

$E_{n}(t)=\frac{n(2 n+1)\left[n(2 n-1)(n-1)^{2}+\delta_{t} \delta(2 n-3)(3 n-2)\right]+\delta^{2} \delta_{t}^{2}(5 n-2)(2 n-3)}{\delta n^{2}\left(4 n^{2}-1\right)(2 n-3)}$.

Note, this form is independent of whether HBIM or RIM is used. The difference appears due to the different expressions for $\delta$. Since $\delta \sim t^{1 / 2}$ in both cases we deduce $E_{n}=e_{n} t^{-1 / 2}$. The minimum error $e_{n}$ and corresponding exponent values for HBIM and RIM are $\left(e_{n}, n\right)=(0.0024,3.584),(0.0029,3.822)$ respectively.

If we apply a cooling condition at the boundary, $u_{x}(0, t)=u(0, t)-1$, then

$$
u=\frac{\delta}{n+\delta}\left(1-\frac{x}{\delta}\right)^{n}
$$

Then $\delta$ satisfies

$$
t=\frac{1}{n(n+1)}\left[\frac{\delta^{2}}{2}+n \delta-n^{2} \ln \frac{n+\delta}{n}\right]
$$

for the HBIM formulation and

$$
t=\frac{1}{(n+1)(n+2)}\left[\delta^{2}+n \delta-n^{2} \ln \frac{n+\delta}{n}\right],
$$

for the RIM. In this case the loss of the simple square root behaviour means that the minimisation process predicts an $n$ which varies with time. In [16] it is shown that $E_{n}$ is a decreasing function of time hence to minimise the error overall the $n$ value corresponding to $t=0$ should be applied. In the limit $t \rightarrow 0$ the condition 
at the boundary become $u_{x}(0, t) \rightarrow-1$ and the appropriate choice of $n$ is clearly that obtained from the constant flux analysis.

2.3. Discussion. So far we have applied the method to the temperature over a semi-infinite domain subject to the three standard boundary conditions. When the temperature at the boundary is fixed the optimal value of $n$ is close to 2 for both HBIM and RIM and the errors are similar with either method. Consequently the usual choice of the HBIM with $n=2$ will give accurate results. With a constant flux boundary condition the HBIM and RIM lead to $n \approx 3.6,3.8$ respectively, again with similar errors. In this case the error decreases from that obtained by using the HBIM with $n=2$ by a factor of more than 30 . In the final case, applying Newtons law of cooling, we find that the optimal $n$ varies with time. Since the maximum error occurs near $t=0$, where the cooling condition reduces to the constant flux condition, the improvement is similar to in the constant flux case. These examples are covered in more detail in [18].

In summary, with a constant temperature boundary condition the RIM with $n=2.218$ gives the most accurate approximation. With constant flux and cooling conditions the HBIM with $n=3.584$ will be most accurate.

\section{Application to Stefan problems}

The HBIM and RIM can provide simple solutions to standard thermal problems. However, their greatest impact is in the field of Stefan problems, where very few analytical solutions are known. We now apply the method of the previous section to three problems involving a change of phase. These highlight different challenges and results for the new method. In the first we consider the classical problem of the melting of a semi-infinite material at solidus. In the second we re-examine the ablation problem of $[3,4,20]$. Finally we look at the classical travelling wave solution.

Note, in the preceding section we restricted our attention to cases where $n \geq 2$ in order to permit solutions that merge smoothly with the constant far field temperature. In the following we do not have such a restriction. In fact we expect non-zero gradients and second derivatives since it is the jump in gradient that drives the interface motion. In the following example we will see that $n \approx 1.8$ provides the best results.

3.1. Melting of a semi-infinite material at the solidus. Consider a semiinfinite material initially at the solidus, occupying $x \geq 0$. At $t=0$ the point $x=0$ is instantaneously heated to a constant temperature greater than the solidus. The material subsequently melts and the position of the melt front is denoted by $x=s(t)$. The non-dimensional problem is described by

$$
\begin{gathered}
\frac{\partial u}{\partial t}=\frac{\partial^{2} u}{\partial x^{2}} \quad 0<x<s(t) \\
u(0, t)=1 \quad \beta(s, t)=0 \quad \beta \frac{d s}{d t}=-\left.\frac{\partial u}{\partial x}\right|_{x=s} .
\end{gathered}
$$

This has the exact solution

$$
u(x, t)=1-\frac{\operatorname{erf}[x /(2 \sqrt{t})]}{\operatorname{erf}(\lambda)}, \quad s(t)=2 \lambda \sqrt{t},
$$


where $\lambda$ satisfies the transcendental equation

$$
\sqrt{\pi} \beta \lambda \operatorname{erf}(\lambda) e^{\lambda^{2}}=1 .
$$

To solve this problem using the HBIM or RIM the approximating function is now expressed in terms of the melt front $s$ (since the material is at the solidus we cannot define a $\delta$ ). We are unable to use a single term model of the form (1) since, for $n>1$, this gives $u_{x}(s, t)=0$ and the melt front is stationary, while for $n<1$ the front velocity is infinite. Consequently, we augment the expression with the simplest usable function, namely a linear function

$$
u=a\left(1-\frac{x}{s}\right)+(1-a)\left(1-\frac{x}{s}\right)^{n} .
$$

This form satisfies $u(0, t)=1, u(s, t)=0$. At $x=s$ the gradient $u_{x}=-a / s$, so for a positive front velocity we require $a>0$.

For the HBIM we integrate the heat equation for $x \in[0, s]$ to give

$$
\frac{d}{d t} \int_{0}^{s} u d x=u_{x}(s, t)-u_{x}(0, t) .
$$

We calculate $u_{x}(0, t)$ and the integral of $u$ using $(26)$. The expression for $u_{x}(s, t)=$ $-\beta s_{t}$ is replaced using the Stefan condition. Note we could also calculate $u_{x}(s, t)$ via the expression (26), this is one of the different formulations discussed in $[12,26]$. We choose to use the Stefan condition to specify $u_{x}$ since this was shown to be the most accurate in [12]. This leads to

$$
s \frac{d s}{d t}=\frac{2(n+1)(a+(1-a) n)}{2+a n-a+2 \beta(n+1)} .
$$

The Stefan condition may be written

$$
s \frac{d s}{d t}=\frac{a}{\beta} .
$$

Assuming $a$ and $n$ are constant gives $s=\sqrt{2 a t / \beta}$. Combining equations (28) and (29) gives a quadratic for $a$

$$
\frac{2(n+1)(a+(1-a) n)}{2+a n-a+2 \beta(n+1)}=\frac{a}{\beta} .
$$

The problem therefore reduces to simply solving (30) for $a$, which in turn determines $s=\sqrt{2 a t / \beta}$ and therefore $u$ through (26). With $a$ and $s$ known in terms of $n$ and $\beta$ we may calculate $E_{n}$.

The RIM leads to

$$
\frac{d}{d t} \int_{0}^{s} x u d x=1+s u_{x}(s, t)=1-\beta s \frac{d s}{d t} .
$$

Evaluating the integral and rearranging

$$
s \frac{d s}{d t}=\frac{3(n+2)(n+1)}{3 a n+a n^{2}-4 a+6+3 \beta(n+1)(n+2)}=\frac{a}{\beta} .
$$

So again we solve a quadratic for $a$ and determine $n$ by minimizing $e_{n}$. 
To calculate $E_{n}$ we need expressions for $u_{t}$ and $u_{x x}$, these are (33)

$$
\frac{\partial u}{\partial t}=\frac{x s_{t}}{s^{2}}\left[a+n(1-a)\left(1-\frac{x}{s}\right)^{n-1}\right] \quad \frac{\partial^{2} u}{\partial x^{2}}=\frac{(1-a) n(n-1)}{s^{2}}\left(1-\frac{x}{s}\right)^{n-2},
$$

which involves the three unknowns $a, n, s$. Using a computer algebra package, such as Maple, it is a simple matter to then calculate $E_{n}$. The expression is cumbersome, so we do not write it down here. However, it is worth noting that as in the example of $\S 2.1$ it has a denominator involving $s s_{t}$. As we will see later $s \propto t^{1 / 2}$ and so $s s_{t}$ is independent of time. The numerator involves $s^{3}$, consequently we may write $E_{n}=e_{n} t^{-3 / 2}$. The minimum value of $e_{n}$ therefore depends on $n$ and $\beta$ and consequently we find that the optimal $n$ varies with $\beta$.

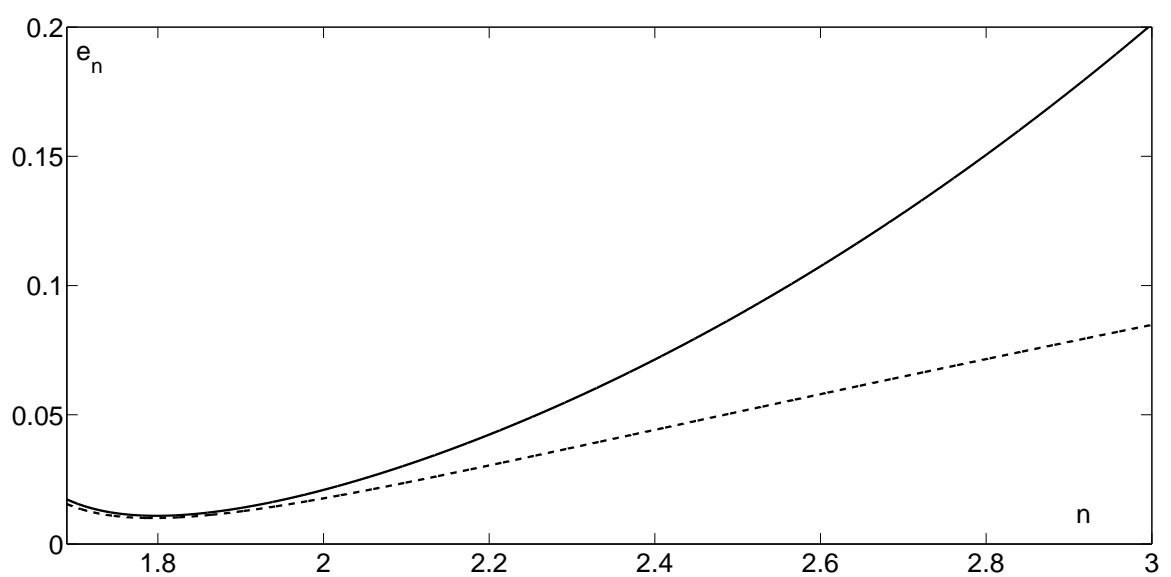

Figure 2. Plot of $e_{n}$ for the melting of a semi-infinite material at solidus with $\beta=1$, for the HBIM formulation (dashed line) and the RIM formulation (solid line).

In Figure 2 we plot $e_{n}$ for $\beta=1$. We allow $n<2$ since for this problem we do not require the temperature to join smoothly to a constant solution. However, singularities exist at $n=1 / 2,3 / 2$. The minimum of $e_{n}$ and corresponding value of $n$ are $(0.01,1.794),(0.01,1.798)$ for the HBIM and RIM formulations respectively. On Figure 3 we show the temperature profiles predicted by the exact and approximate methods at $t=0.1$ (again for $\beta=1$ ). The HBIM solution is the dotted line slightly above the solid line of the exact solution. The dashed line of the RIM solution shows excellent agreement, and is only distinguishable from the exact solution over a small region. In this case, since we can obtain an expression for $e_{n}$ the appropriate value of $n$ to minimise $E_{n}$ will not depend on time and we expect the error in temperature prediction, $E_{n}(t)$, to decrease with time. The variation of $n$ with $\beta$ is relatively small for realistic $\beta$; for $\beta \in[0.7,50]$ we find $n$ decreasing in the range $[1.804,1.765]$ for HBIM and $[1.809,1.769]$ for RIM.

The most important quantity of the Stefan problem is the position of the moving front; this is shown in Figure 4 for $\beta=1$. Five curves are plotted, 


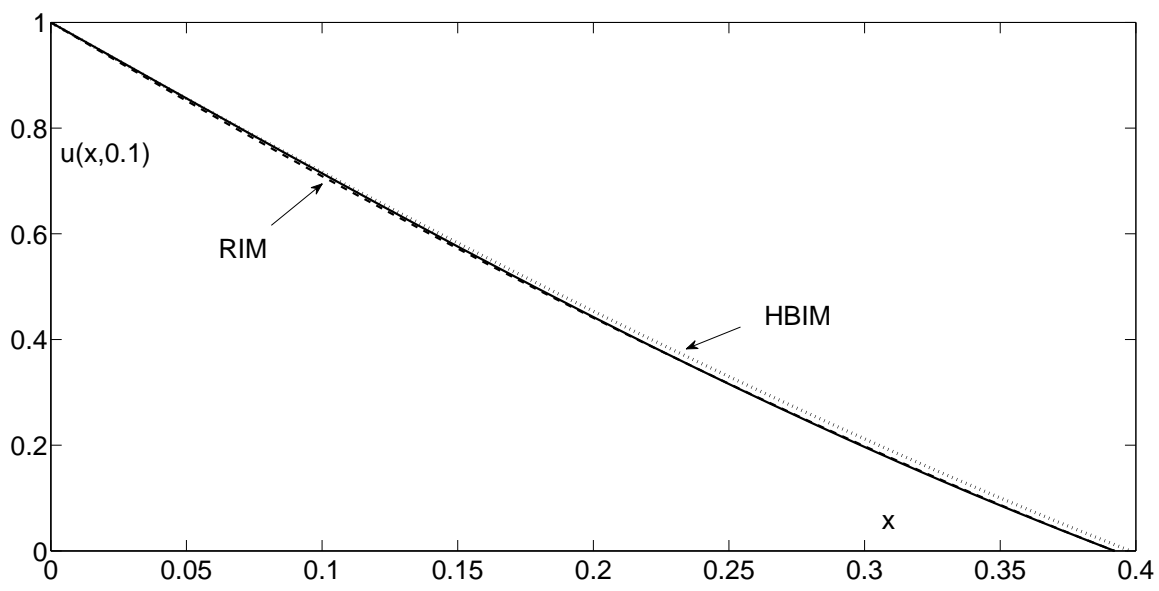

Figure 3. Comparison of $u(x, t)$ at $t=0.1$ for exact (solid line), RIM (dashed) and HBIM (dotted) solutions.

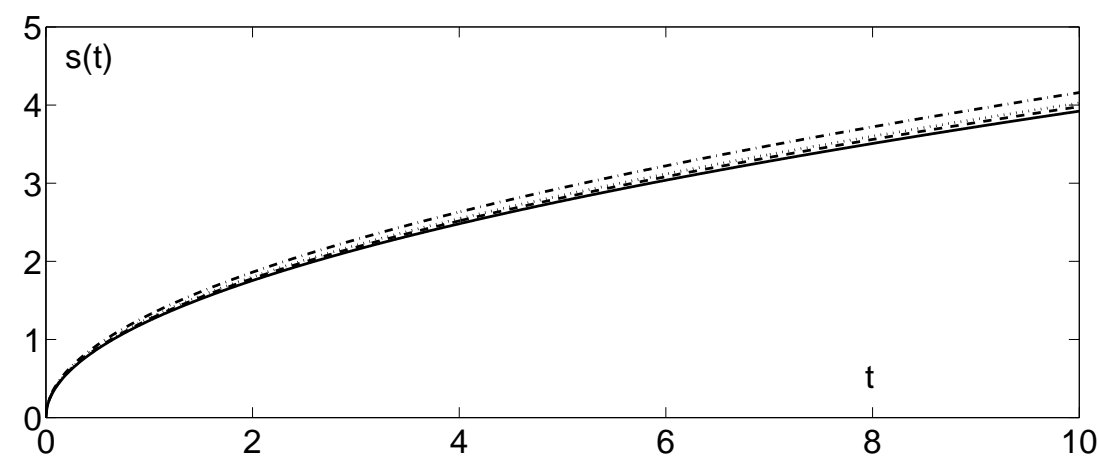

FIGURE 4. Comparison of $s(t)$ predicted by exact and RIM with $n=1.798$ (solid line), HBIM with $n=1.794$ (dashed) and HBIM with $n=2,3$ (dotted and dash-dot lines) solutions.

although only four are visible. The solid line incorporates both the RIM and exact solutions which are indistinguishable, the dashed line is the HBIM solution with $n=1.794$. The two lines above this are the standard HBIM solutions with $n=2$ and 3 , shown as dotted and dash-dot lines. The close correspondence between the optimal RIM and the exact solution may be inferred by comparing the expressions for $s$, where we see that for the exact solution $s=2 \lambda \sqrt{t}$, for the approximate solutions $s=\sqrt{2 a t / \beta}$. The percentage error between $2 \lambda$ and $\sqrt{2 a / \beta}$ for the optimized RIM solution is $0.02 \%$. The percentage errors for optimized HBIM and using $n=2,3$ are $1.5 \%, 2.65 \%$ and $6.1 \%$, that is, the reduction in error gained by using the optimized RIM over the HBIM with $n=3$ is a factor of over 300 . 


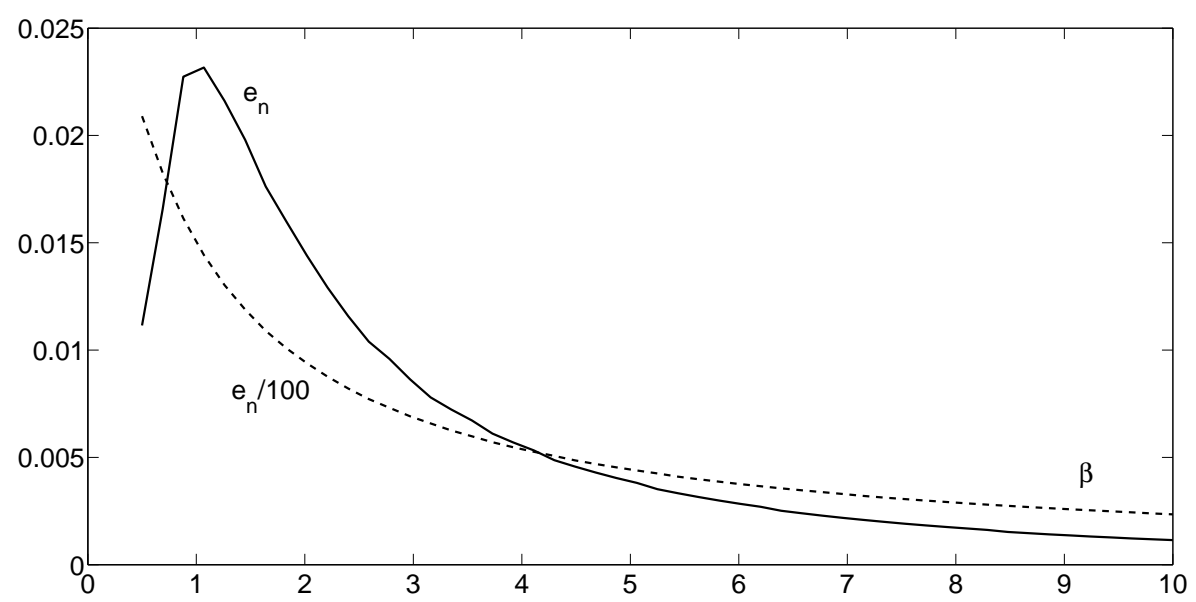

FIGURE 5. Variation of $e_{n}$ with $\beta$ for the RIM (solid) and $e_{n} / 100$ for HBIM (dashed) formulation.

It is well-known that the accuracy of HBIM and RIM solutions depends on the value of $\beta[12,26]$. The actual value of $\beta$ is given by $\beta=\left(\rho_{s} / \rho_{l}\right) L_{m} /\left(c_{l} \Delta u\right)$, where $\rho$ represents the density of the solid or liquid phase, $L_{m}$ is the latent heat of melting, $c_{l}$ the heat capacity of the liquid and $\Delta u$ the temperature drop $u_{s}-u_{i}$. For an ice-water system appropriate values for these constants may be found in $[15,17]$. If we assume a maximum temperature difference $\Delta u=100$ then $\beta \geq 0.71$. Alexiades \& Solomon [1] state that for water and paraffin wax typically $\beta \in[1,10]$, for metals $\beta \in[0.1,1]$.

For the current problem the HBIM error, whilst small, is two orders of magnitude larger than the RIM error, consequently on Figure 5 we show the minimum value of $e_{n}$ for the RIM and $e_{n} / 100$ for the HBIM formulations with $\beta \in[0.5,10]$. The error for the RIM peaks close to $\beta=1$ (with a maximum around 0.024 ) and then steadily decreases, at $\beta=10$ the error is around 0.001 . The example we show in Figure 4 , with $\beta=1$, clearly shows excellent agreement with the exact solution but, since the error is largest $\beta \approx 1$, the approximate solution actually shows close to the worst agreement with the exact solution that we could present. In fact one reason for plotting solutions for this value of $\beta$ is that for larger $\beta$ it is difficult to distinguish any of the curves.

3.2. Ablation due to a constant flux. Unlike the thermal problems of $\S 2$ the previous example had no $\delta(t)$, since for $x>s$ the temperature $u=0$. So in the following example we choose a case involving both $s(t)$ and $\delta(t)$.

Consider a material occupying $x \geq 0$, initially at temperature $u(x, 0)=0$. At time $t=0$ a constant flux is applied at $x=0$, this heats the material up to the ablation temperature, $u=1$, after which the ablated material is removed and the remaining material occupies $x \geq s(t)$. Consequently this problem occurs in two distinct stages. In the first the material has a fixed boundary and proceeds as the constant flux example of $\S 2$. In the second stage the boundary $s(t)$ moves 
while the boundary temperature remains at the ablation temperature. Braga \& Mantelli $[3,4]$ study this problem using $n=\pi /(4-\pi)$ in the initial heating up phase and subsequently $n=7$ during ablation. Mitchell \& Myers [13] study the same problem and take $n=4$ in both cases. They also show that switching the exponent once ablation starts is only valid provided the second exponent is not greater than the initial exponent. If this is violated then for a small period the ablated material returns. Comparison with numerical results in [13] shows that $n=4$ appears to give the best results for small times after ablation commences, $n=7$ is subsequently better but then the error grows. These results indicate that $n=n(t)$. This will be confirmed in our subsequent analysis.

The initial heating up phase is simply the constant flux case of $\S 2.2$. This ends at time $t_{1}$ when the boundary reaches the ablation temperature, which we have conveniently scaled to be $u(0, t)=1$. With the temperature given by equation (15) we find an expression for $\delta_{1}=\delta\left(t_{1}\right)=n$. We require $\delta_{1}$ to provide an initial condition for $\delta$ in the ablation stage. Taking the relations for $\delta(t)$, equations $(16,17)$, means that for the HBIM formulation $t_{1}=n /(n+1)$. For the RIM $t_{1}=3 n^{2} /(2(n+1)(n+2))$.

Once ablation starts the material occupies $x \geq s(t)$. At $x=s$ we have $u=1$, while the Stefan condition is

$$
\beta \frac{d s}{d t}=1+\left.\frac{\partial u}{\partial x}\right|_{x=s} .
$$

For the approximate solutions we also set $u=u_{x}=0$ at $x=\delta$ and find a temperature of the form

$$
u=\left(\frac{\delta-x}{\delta-s}\right)^{n}
$$

To apply the HBIM we integrate over $x \in[s, \delta]$ to find

$$
\frac{d}{d t} \int_{s}^{\delta} u d x+\frac{d s}{d t}=-\left.\frac{\partial u}{\partial x}\right|_{x=s} .
$$

Substituting for $u$ and evaluating the integral leads to

$$
\frac{d \delta}{d t}+n \frac{d s}{d t}=\frac{n(n+1)}{\delta-s} .
$$

The Stefan condition provides a second equation for the unknowns

$$
\beta \frac{d s}{d t}=1-\frac{n}{\delta-s} .
$$

Eliminating the denominator $\delta-s$ in the two equations results in an equation that can be integrated immediately

$$
\delta+(n+(n+1) \beta) s=(n+1)\left(t-t_{1}\right)+\delta_{1} .
$$

This allows us to eliminate $\delta$ in one of the differential equations and integrate a single equation numerically (for a given $n$ ). 


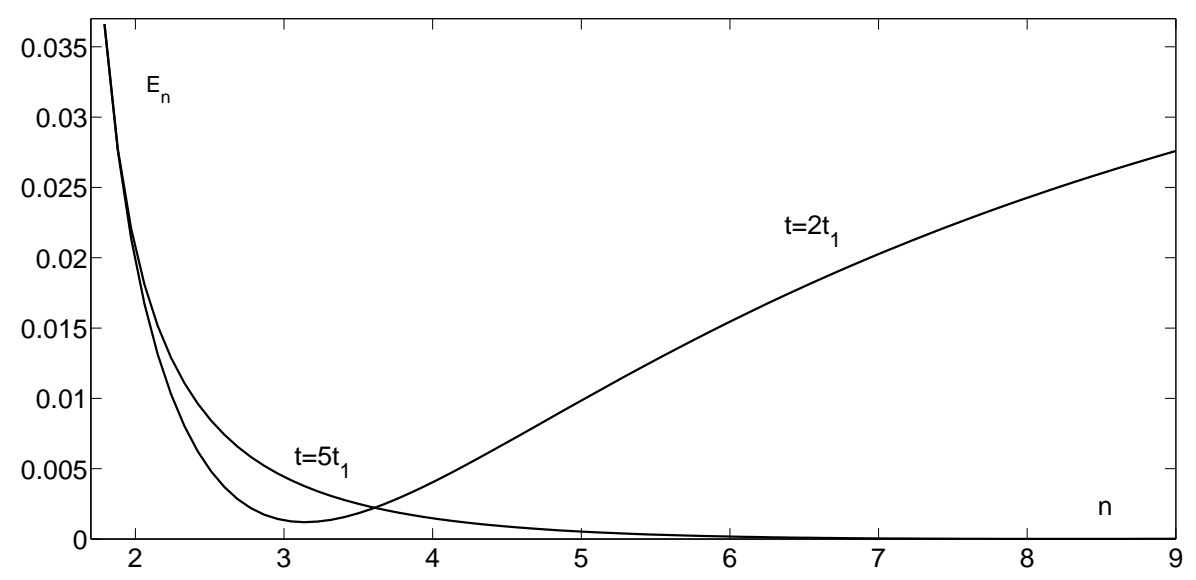

Figure 6. Plot of $E_{n}$ for $t=2 t_{1}, 5 t_{1}$.

When calculating $E_{n}$ we note that the temperature is defined over $x \in[s, \delta]$ so the integral must be evaluated over this domain (rather than $x \in[0, \delta]$ as in the previous problems). The necessary derivatives are

$\frac{\partial u}{\partial t}=n \delta_{t} \frac{(\delta-x)^{n-1}}{(\delta-s)^{n}}-n\left(\delta_{t}-s_{t}\right) \frac{(\delta-x)^{n}}{(\delta-s)^{n+1}} \quad, \quad \frac{\partial^{2} u}{\partial x^{2}}=n(n-1) \frac{(\delta-x)^{n-2}}{(\delta-s)^{n}}$.

$E_{n}$ is then calculated numerically after the $\delta$ 's have been found. Given that there is no closed form solution for $s$ and $\delta$ it seems unlikely we can express $E_{n}=e_{n} t^{\alpha}$. To demonstrate this in Figure 6 we plot the numerical solution for $E_{n}$ for two times, $t=2 t_{1}, 5 t_{1}$ and find that the value of $n$ that minimizes the function changes from 3.136 to 8.154. So $n$ is an increasing function of time. However, an important feature of this solution is that although the optimal value of $n$ increases with time the error decreases. For example, at $2 t_{1}$ the best value is $n=3.136$ with $E_{n}\left(2 t_{1}\right)=0.001$. At $t=5 t_{1}$, even though the error is a minimum at $n=8.154, E_{n}\left(5 t_{1}\right) \approx 10^{-5}$. In practice we can obtain good results by minimizing the error for small time (so keeping $n$ low) and for large times any value of $n$ greater than say 3.5 will retain a small error. As noted in [13] we cannot increase the value of $n$ from pre- to post-ablation. Given that the optimal $n$ from the pre-ablation is $n=3.584$ then we can safely use this for all time knowing that it provides the best solution for pre-ablation and a small error for post-ablation. In Figure 7 we show the evolution of $s(t)$ for the two cases $n=3.584,8.154$ and compare these with a numerical solution obtained using the method described in [13]. The numerical solution is shown as a solid line, with $n=3.584$ as a dashed line and $n=8.154$ as a dotted line. An improved numerical method for dealing with single phase Stefan problems, such as ablation is described in [14]. We only take a small variation in time, since this allows us to see the difference in curves more easily. If we take the final time for example as $10 t_{1}$ then the larger scale results in three barely distinguishable curves. Even with the small time range 
the curves are clearly very similar and so in Figure $7 \mathrm{~b}$ we show a close-up of the early time solution, where it may be seen that for $n=8.154$ the melt position initially becomes negative and then stays below the exact solution, for larger times it approaches the numerical solution. The solution for $n=3.584$ is almost indistinguishable from the exact solution for small times but moves above the numerical solution as time increases. If we take even larger times the curves do not diverge from the numerical one, a fact that could be inferred from the plot of $E_{n}$ which decreases with time.
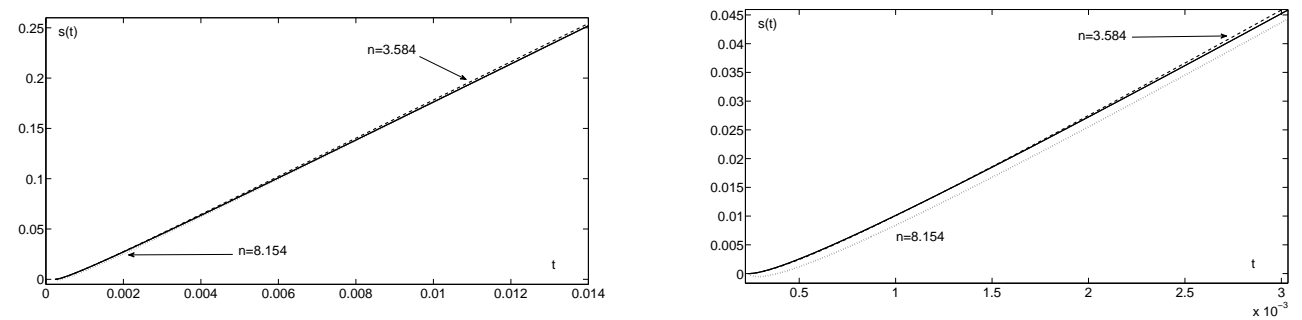

Figure 7. a) Plot of $s(t)$ calculated by numerical solution (solid line) and HBIM with $n=3.584$ (dashed) and $n=8.154$ (dotted), $t \in\left[t_{1}, 4 t_{1}\right]$ b) blow-up for small times

The RIM formulation leads to $\delta_{1}=n, t_{1}=3 n^{2} /(2(n+1)(n+2))$ and

$$
\frac{d}{d t} \int_{s}^{\delta} x u d x+s \frac{d s}{d t}=\frac{n \delta}{\delta-s}+1-n
$$

which replaces equation (36). Again we find that the error depends on time and conclude that the optimal $n$ comes from pre-ablation. Taking $n=3.822$ results in a melt rate almost indistinguishable from the HBIM curve shown in Figure 7.

3.3. Example with growth rate $\propto t$ : a cautionary tale. We now look for a travelling wave solution in terms of the variable $\eta=x-c t$. The result is a solution of the form

$$
u(x, t)=\beta\left(e^{-c(x-c t)}-1\right), \quad s(t)=c t .
$$

Consequently we can look for solutions using the approximate method subject to the following time-dependent boundary conditions at $x=0$ :

$$
u(0, t)=\beta\left(e^{c^{2} t}-1\right) .
$$

So, for this example the melt front moves with a constant velocity. In order to achieve this an exponentially increasing temperature is applied at the origin. One can then view this example as having mathematical interest but, except for small times, it is physically unrealistic. Caldwell \& Kwan [5] study this problem with $\beta=c=1$. They also study a number of other problems and compare various numerical methods and a linear discretised HBIM. However, they do not present results for the HBIM approach to this problem, stating it is useful for situations with a constant boundary condition but difficult to extend to time-dependent ones. Mitchell \& Myers [12] find solutions using HBIM, RIM and a modified 
RIM up to non-dimensional time $t=1$. In each case the cubic approximating function is more accurate than the quadratic.

As in $§ 3.1$ we use an approximating function with a linear term

$$
u=a\left(1-\frac{x}{s}\right)+b\left(1-\frac{x}{s}\right)^{n}
$$

where

$$
b=\beta\left(e^{c^{2} t}-1\right)-a .
$$

This satisfies the boundary conditions at $x=0, s(t)$. The HBIM gives

$$
\frac{d}{d t}\left[\left(\frac{a}{2}+\frac{b}{n+1}\right) s\right]=-\beta \frac{d s}{d t}+\left(\frac{a}{s}+\frac{b n}{s}\right)
$$

and the Stefan condition is

$$
\frac{d s}{d t}=\frac{a}{s}
$$

So we have two equations for two unknowns, $a$ and $s$ (note we cannot assume $a$ is constant for this problem).

The RIM formulation leads to

$$
\frac{d}{d t} \int_{0}^{s} x u d x=u(0, t)+s u_{x}(s, t)
$$

and so

$$
\frac{d}{d t}\left[\left(\frac{a}{6}+\frac{b}{(n+1)(n+2)}\right) s^{2}\right]=b .
$$

After minimizing $E_{n}$ again we find that $n$ varies with time. However, in this case the error grows in time. Given that the temperature at $x=0$ increases exponentially this is not too surprising. Consequently for early times we can find relatively accurate solutions, but as time increases the accuracy decreases. For example, when the end time $t=1$, for the RIM formulation, we find $n=2.39$ and $E_{n}(1)=0.036$, when $t=2, n=3.05$ and $E_{n}(2)=0.44$, with $t=4, n=5.1$ and $E_{n}(4)=11.43$. So the error grows rapidly as $t$ increases (presumably exponentially). The HBIM approach has a similar increase in the error. Consequently one should only use this approach for small times. In Figure 8 we show two plots for $s(t)$ up to times $t=1$ and 4. In Figure 8a) we take $n=2.386$ and 2.392 for the RIM and HBIM results respectively, corresponding to errors of $0.036,0.04$. The small value of $E_{n}$ indicates that the results are very close to the exact solution. We could use these values of $n$ for smaller time calculations and obtain errors of this magnitude or we could reduce the error by recalculating $n$. However, for larger times we will need larger values of $n$ to prevent the error from increasing too rapidly. In Figure $8 \mathrm{~b}$ ) we take $n=5.1$ and 6.2 (the values calculated for $t=4$ ), corresponding to minimum errors of 11.4, 9.4. Now the large value of $E_{n}$ indicates that the results are not very accurate. As time increases our ability to track the moving front deteriorates and the error grows. 

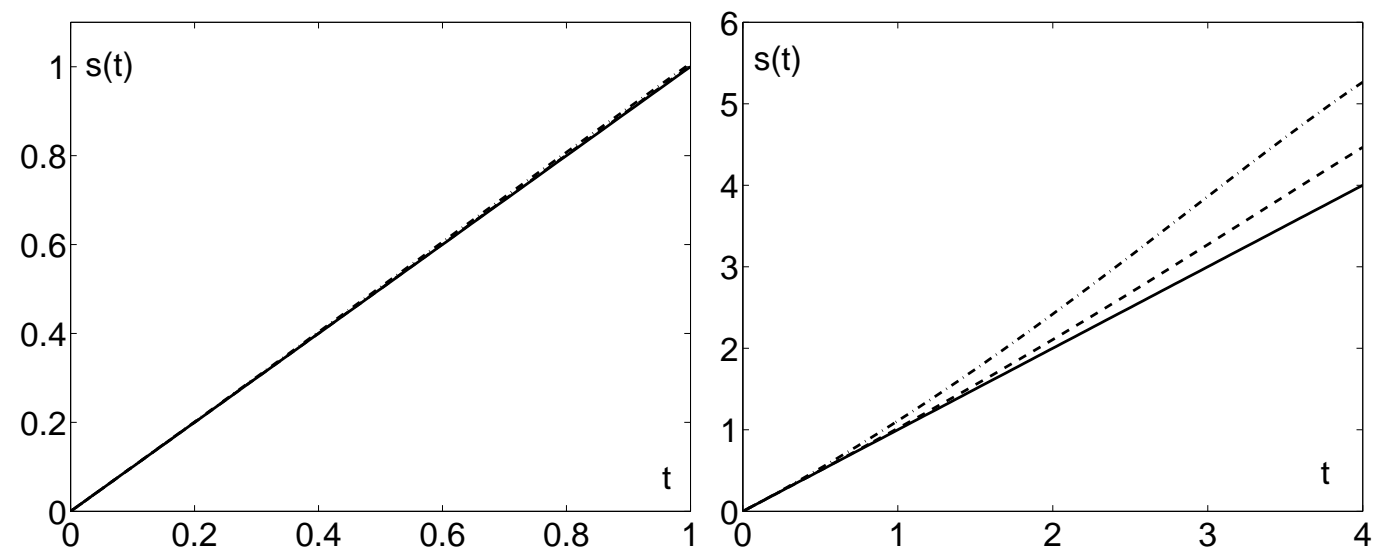

Figure 8. a) Plot of $s(t)$ up to a) $t=1, b) t=4$ solid line is the exact solution, dashed is the RIM and dash-dot the HBIM

3.4. Melting with a time-dependent flux. In all of the previous examples we have dealt with problems where either an exact solution is known, or a numerical solution can easily be calculated. We now construct and solve a problem without resorting to a known solution.

Consider the situation described in $\S 3.1$, but with the boundary condition $u(0, t)=1$ changed to $u_{x}(0, t)=-1 / s(t)$. We choose this form for two reasons. Firstly, the expression for $u$ is relatively simple with this choice and secondly, it is difficult to analyse either analytically or numerically.

Taking a profile of the form (26) and applying $u_{x}(0, t)=-1 / s(t)$ gives

$$
u=\left(1-n a_{n}\right)\left(1-\frac{x}{s}\right)+a_{n}\left(1-\frac{x}{s}\right)^{n} .
$$

The heat balance integral is

$$
\frac{d}{d t}\left[\left\{\beta+\left(\frac{1-n a_{n}}{2}\right)+\frac{a_{n}}{n+1}\right\} s\right]=\frac{d}{d t}\left(\gamma_{1} s\right)=\frac{1}{s}
$$

where $\gamma_{1}$ represents the terms in curly brackets, hence $s=\sqrt{2 t / \gamma_{1}}$. From the Stefan condition $s=\sqrt{2\left(1-n a_{n}\right) t / \beta}$. Equating these two expressions provides a quadratic for $a_{n}$ in terms of $n, \beta$. Using Maple we find $E_{n}=e_{n} t^{-3 / 2}$ and consequently determine $n=1.718$ by minimising $e_{n}$ (with a minimum value $\left.e_{n}=0.0065\right)$. The small value of $e_{n}$ indicates that this solution will be very accurate.

For the RIM formulation

$$
\frac{d}{d t}\left[\left\{\frac{1-n a_{n}}{6}+\frac{a_{n}}{(n+1)(n+2)}\right\} s^{2}\right]=\frac{d}{d t}\left(\gamma_{2} s^{2}\right)=n a_{n}
$$

and $s=\sqrt{a_{n} t / \gamma_{2}}$. In this case $e_{n}=0.0072$, so we expect the RIM to be slightly less accurate, and $n=1.718$ when $\beta=1$. We do not present a comparison of 
results for this problem, since clearly $s$ takes the standard square root form (see figure 4) and the two sets of results are very similar.

3.5. Discussion. Four Stefan problems have been discussed in this section. The results can be summarized as follows:

(1) Fixed temperature boundary condition: both HBIM and RIM are optimized for $n \approx 1.8$. The error obtained through the HBIM with $n=2$ is also relatively small (since the choice $n=2$ is close to the optimal value). However, even with this small variation in $n$, the error in the growth rate reduces by a factor of 130 when using the optimized RIM rather than the HBIM with $n=2$. It is also worth noting that the optimum $n$ varies weakly with $\beta$ (around a $2 \%$ variation for realistic $\beta$ ).

(2) Ablation due to constant flux: we find $n=n(t)$ and it appears best to employ the HBIM with $n \approx 3.6$ throughout the calculation. Using this value will decrease the error in the position of the moving front by a factor of around 20 when compared to the HBIM with $n=2$.

(3) Travelling wave: $n$ is a function of time and the error grows with $t$. In this case heat balance methods should only be trusted for small times.

(4) Flux $\propto 1 / s$ : the best method here is to use the HBIM with $n \approx 1.7$. When $\beta=1$ the error $e_{n} \approx 0.0065$. Using $n=2$ gives $e_{n} \approx 0.013$ (i.e. the error is doubled).

\section{Conclusion}

The aim of this work was to determine the optimal value of the exponent $n$ for certain thermal and phase change problems, where optimal has been defined in the sense of the Langford criteria of minimising the square of the heat equation. No doubt other criteria could be used, however Langford's method has the advantage over standard error measures in that it does not require knowledge of an exact solution. A definite disadvantage is observed in the cases where we could write $E_{n}(t)=e_{n} t^{\alpha}$. The value of $\alpha$ was always negative hence as $t \rightarrow 0, E_{n} \rightarrow \infty$ even though the approximation remains good. So, $E_{n}$ can provide a rather poor indication of the accuracy. The value of $e_{n}$ appears to be a more reliable indicator, but this does not exist for every problem. Perhaps then some normalisation of $E_{n}$ would resolve the issue, i.e. we define $E_{n}=\int\left(u_{t}-u_{x x}\right)^{2} d x / \int u_{x x}^{2} d x$ for example.

We have demonstrated the method with a standard thermal problem and four Stefan problems. For the thermal problem and the first two Stefan problems the agreement with exact or numerical solutions was excellent. The third case, with an exponentially growing heat source at the origin proved to be amenable to the method only for small times. With the fourth example the value of $e_{n}$ indicated that the solution was accurate.

In Table 1 we summarise the cases and the value of $n$ that should be used to obtain the best results for three standard thermal problems and also the three Stefan problems where the method worked well. Note, although we have worked throughout with non-dimensional quantities the results hold for the equivalent dimensional systems. For most situations the error when using HBIM or RIM is similar, provided the correct value of $n$ is used. The exception is the classical problem of melting a semi-infinite material at the solidus. In this case the RIM should be used, which shows errors two orders of magnitude less than HBIM. 
The optimal value of $n$ depends on the parameter $\beta$, however for realistic $\beta$ the variation is small. We have quoted the values for $\beta=1$ in the Table. For the ablation problem we choose $n$ from the pre-ablation problem. The maximum error occurs just after ablation begins, at time $t \approx t_{1}$ : in the table we show the error when $t=1.001 t_{1}$. If we were to use the value of $n$ that minimizes $E_{n}$ then the maximum error would be $\mathcal{O}(0.03)$ in both cases, however, then we must vary $n$ as the ablation progresses.

For the problem where $u(0, t) \sim e^{t}$ we cannot in general define an optimal $n$. Instead the best strategy appears to be to determine $n$ for the final time and use this throughout the calculation. However, the error will grow with $t$ and so this result should not be assumed accurate for large times.

\begin{tabular}{|l|c|c|c|c|}
\hline Boundary condition & HBIM $n$ & RIM $n$ & Error, HBIM & Error, RIM \\
\hline \multicolumn{5}{|c|}{ Thermal problems } \\
\hline Fixed temperature & 2.235 & 2.218 & $e_{n}=0.0169$ & $e_{n}=0.0167$ \\
Constant flux & 3.584 & 3.82 & $e_{n}=0.0024$ & $e_{n}=0.0029$ \\
Cooling condition & $n \leq 3.58$ & $n \leq 3.82$ & $E_{n}(t)<0.02$ & $E_{n}(t)<0.02$ \\
\hline \multicolumn{5}{|c|}{ Stefan problems } \\
\hline \begin{tabular}{l|c|c|c|c|} 
semi- $\infty$ at solidus, \\
fixed temp \\
semi- $\infty$ at solidus,
\end{tabular} & 1.716 & 1.724 & $e_{n}=0.0066$ & $e_{n}=0.0069$ \\
$u_{x}(0, t)=-1 / s^{\star}$ & 3.584 & 3.82 & $E_{n}(t)<0.11$ & $E_{n}(t)<0.13$ \\
$\begin{array}{l}\text { Ablation, constant } \\
\text { flux }\end{array}$ & 3.8 & $e_{n}<2$ & \\
\hline
\end{tabular}

TABLE 1. Optimal values of $n$ for different thermal problems

( ${ }^{\star}$ requires extra term in temperature expression, see $§ 3.1$ )

We have not carried out an exhaustive study of applications of the integral methods, since there are too many of these. However, we have illustrated the application of the new method to typical problems and hope this is sufficient to guide the user in other applications. The majority of examples shown in this paper were compared to existing solutions. This was done merely for the purpose of demonstrating the accuracy of the method. In the final example we have no analytical or numerical solution and so must trust to the method. The small value of $e_{n}$ indicates that the chosen polynomial is a good approximation. In general this is how the method would ideally be used, namely, one merely finds the value of $n$ that minimises $E_{n}$ and uses this throughout the calculation, safe in the knowledge that the approximation will not improve with a different choice of $n$.

One of the goals of this paper was to remove the ambiguity in the choice of approximating function. For thermal problems this seems to have been achieved. For Stefan problems some ambiguity remains. Two of the cases studied required 
the addition of an extra term in the temperature expression. We used a linear function for simplicity; it drops out of the expression for $u_{x x}$ required in the error calculation. Other terms could be employed. When the optimal $n$ varies with time we have chosen the value corresponding to $t=0$ (or $t_{1}$ if melting is not immediate). This seems appropriate when $E_{n}$ is a decreasing function of time, so we attempt to minimize the maximum error. When $E_{n}$ grows with time it is not so clear what is the best choice. We took the value of $n$ to minimize the error at the final time. This choice may be open to debate.

\section{ACKNOWLEDGEMENTS}

The author would like to acknowledge the support of the Institute of Applied Mathematics at the University of British Columbia, where this work was completed.

\section{REFERENCES}

[1] V. Alexiades and A.D. Solomon. Mathematical modeling of melting and freezing processes. Hemisphere Publishing Corporation, 1993.

[2] A. Antic and J.M. Hill. The double-diffusivity heat transfer model for grain stores incorporating microwave heating. Appl. Math. Modelling, 27: 629-647, 2003.

[3] W. F. Braga, M. B. H. Mantelli, and J. L. F. Azevedo. Approximate analytical solution for one-dimensional ablation problem with time-variable heat flux. In AIAA Thermophys. Conference, June 2003.

[4] W. F. Braga, M. B. H. Mantelli, and J. L. F. Azevedo. Approximate analytical solution for one-dimensional finite ablation problem with constant time heat flux. In AIAA Thermophys. Conference, June 2004.

[5] J. Caldwell and Y. Y. Kwan. Numerical methods for one-dimensional Stefan problems. Comm. Num. Methods Eng., 20: 535-545, 2004.

[6] H. S. Carslaw and J. C. Jaeger. Conduction of Heat in Solids, 2nd Edition. Oxford University Press, London, 1959.

[7] T. R. Goodman. The Heat-Balance Integral and Its Application to Problems Involving a Change of Phase. Trans. ASME, 80: 335-342, 1958.

[8] J. Hristov. Research note on a parabolic heat-balance integral method with unspecified exponent: An entropy generation approach in optimal profile determination. Thermal Sci., 13(2):49 - 59, 2009. DOI: 10.2298/TSCI0902049H.

[9] J. Hristov. The heat-balance integral method by a parabolic profile with unspecified exponent: analysis and exercises. Thermal Sci., 13(2):27 - 48, 2009. DOI: 2298/TSCI0902027H.

[10] S. Kutluay, A. S. Wood, and A. Esen. A heat balance integral solution of the thermistor problem with a modified electrical conductivity. Appl. Math. Modelling, 30: 386-394, 2006.

[11] D. Langford. The heat balance integral method. Int. J. Heat \& Mass Trans., 16: 2424-2428, 1973.

[12] S. L. Mitchell and T. G. Myers. The application of standard and refined heat balance integral methods to one-dimensional Stefan problems. To appear SIAM Review.

[13] S. L. Mitchell and T. G. Myers. A heat balance integral method for one-dimensional finite ablation. AIAA J. Thermophys. \& Heat Trans., 22(3): 508-514, 2008.

[14] S. L. Mitchell and M. Vynnycky. Numerical methods for one-dimensional single-phase Stefan problems. To appear Appl. Math. Comp.

[15] T. G. Myers. Extension to the Messinger model for aircraft icing. AIAA J., 39(2), 2001.

[16] T. G. Myers. Optimizing the exponent in the heat balance and refined integral methods. Int. Comm. Heat \& Mass Trans., 2008. DOI:10.1016/j.icheatmasstransfer.2008.10.013. 
[17] T. G. Myers and J. P. F. Charpin. A mathematical model for atmospheric ice accretion and water flow on a cold surface. Int. J. Heat \& M Mass Trans., 47, 2004.

[18] T. G. Myers and S. L. Mitchell. Application of the heat balance and refined integral methods to the Korteweg-de Vries equation. Thermal Sci., 13(2):113 - 119, 2009. DOI: 10.2298/TSCI0902113M.

[19] T. G. Myers, S. L. Mitchell, and G. Muchatibaya. Unsteady contact melting of a rectangular cross-section material on a flat plate. Phys. Fluids, 20, 2008. DOI:10.1063/1.2990751.

[20] T. G. Myers, S. L. Mitchell, G. Muchatibaya, and M. Y. Myers. A cubic heat balance integral method for one-dimensional melting of a finite thickness layer. Int. J. Heat 83 Mass Trans., 50: 5305-5317, 2007.

[21] J. R. Ockendon, S. D. Howison, A. A. Lacey, and A. Movchan. Applied Partial Differential Equations. Oxford University Press, 1999.

[22] N. Sadoun, E-K. Si-Ahmed, and P. Colinet. On the refined integral method for the onephase Stefan problem with time-dependent boundary conditions. App. Math. Modelling, 30: 531-544, 2006.

[23] N. Sadoun and E.K. Si-Ahmed. A new analytical expression of the freezing constant in the stefan problem with initial superheat. Numer. Meth. Therm. Probl., 9:843854, 1995.

[24] H. Schlichting. Boundary layer theory. Springer, 8th edition, 2000.

[25] M. J. Spearpoint and J. G. Quintiere. Predicting the piloted ignition of wood in the cone calorimeter using an integral model effect of species, grain orientation and heat flux. Fire Safety J., 36(4): 391-415, 2001.

[26] A. S. Wood. A new look at the heat balance integral method. App. Math. Modelling, 25: 815-824, 2001.

Centre de Recerca Matemàtica

UAB, SCIENCE FACUlTy

08193 Bellaterra, Barcelona, Spain 